\title{
Polarization Distributions in Isotropic, Stretched or Annealed PVDF Films
}

\author{
E. Bihler, K. Holdik ${ }^{\star}$ and W. Eisenmenger \\ Physikalisches Institut, Universität Stuttgart, \\ Pfaffenwaldring 57,7000 Stuttgart 80 \\ *present address: SEL AG, ZT/FZWM, Lorenzstraße 10, 7000 Stuttgart 40
}

\section{Introduction}

The spatial distribution of the piezoelectric coefficient in polarized PVDF has been investigated by several authors using different methods $/ 1-6 /$. In addition also the time development of the polarization distribution in PVDF under external electric fields was studied for a variety of commercially available PVDF films from different suppliers using the piezoelectric pressure step (PPS) technique $/ 7 /$. For a field strength ranging from 0.5 to $1 \mathrm{MV} / \mathrm{cm}$, samples from different suppliers developped either i) a central polarization zone with no piezoeffect in the boundary regions close to the film surface or ii) a polarization maximum close to the positive charging electrode (anode) without polarization from about the centre of the film to the negative electrode. Since sample inhomogeneities can be excluded, the development of polarization zones are to be explained by charge injection and trapping /3,9/.

The different behaviour i) and ii) in principle must be attributed either to possible differences in the general chemical composition of the films of different suppliers or to differences in the crystallite structure. So far it was found $/ 11 /$ that the central polarization zone i) appeared in films with a finite content of $\beta$-form crystallites, whereas the polarization maximum at the positive electrode ii) appeared for pure $\alpha$-material. In order to check the possible influence of the crystallite structure independently from the chemical sample composition, we investigated the polarization distributions for the same material as received first with dominant $\alpha$-crystallite content. then after biaxially stretching in order to increase the $\beta$-crystallite content and rinaily after annealing again in order to reduce the $\beta$-crystallite content.

\section{Experimental Technique}

The spatial distribution of the polarization in PVDF films was measured at room temperature with the PPS-method $/ 3,7 /$. In order to investigate the time development under external fields a thin insulating PET-film covering an evaporated aluminium electrode was inserted between sample and the measuring electrode. This additional polymer acts as a broadband coupling capacitor to the measured displacement current. It does not influence the shape of the signal.

The crystallite phase composition of the PVDF films was determined by IR absorption. The ratio between the relative absorption at $530 \mathrm{~cm}^{-1}\left(\alpha\right.$-crystallites) and $510 \mathrm{~cm}^{-1}$ ( $\beta$-crystallites) is taken as a standard for the $\alpha / \beta$-phase content (according to $/ 8 /$ ). 


\section{Results}

A commercial PVDF film ("Dyffor 2000", Dynamit-Nobel, Troisdorf, FRG) with almost all crystallites of $\alpha$ type was poled at room temperature. The film was poled $1 \mathrm{~h}$ at a field strength of $0.5 \mathrm{MV} / \mathrm{cm}$ and afterwards kept under zero voltage conditions for another hour. The piezo profile in Fig. 1 shows a sharp peak located closely to the anode.

Fig. 1:

Isotropic PVDF film, thickness $110 \mu \mathrm{m}$ poled $1 \mathrm{~h}$ at $0.5 \mathrm{MV} / \mathrm{cm}$.

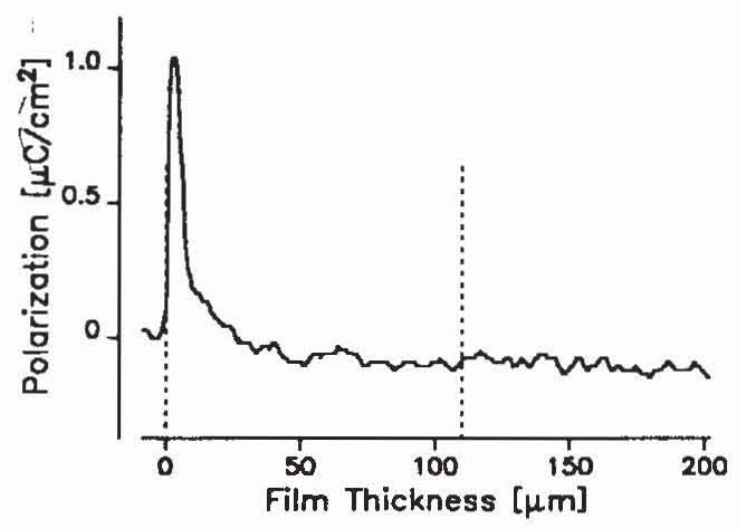

The same material then was stretched at a temperature of $150{ }^{\circ} \mathrm{C}$ with a thickness reduction to about $20 \%$ of its original value. The $\alpha / \beta$ phase ratio of the stretched film was 3.9. The time development of the polarization profiles of the stretched film is shown in Fig.2a for a field strength of $0.6 \mathrm{MV} / \mathrm{cm}$. The development starts always with a homogeneous profile caused by the external field. The signal then begins to change its shape indicating homo charge injection from both electrodes, the build up of an almost central polarization zone with polarization free film boundaries and trapping of injected charges at the boundaries of the polarization zone. (The same polarization development was already observed in stretched films as received with finite $\beta$ crystallite content $/ 7 /$.)

Fig. 2a:

Stretched film, thickness $20 \mu \mathrm{m}$, $\alpha / \beta$ phase ratio 3.9 field strength $0.6 \mathrm{MV} / \mathrm{cm}$

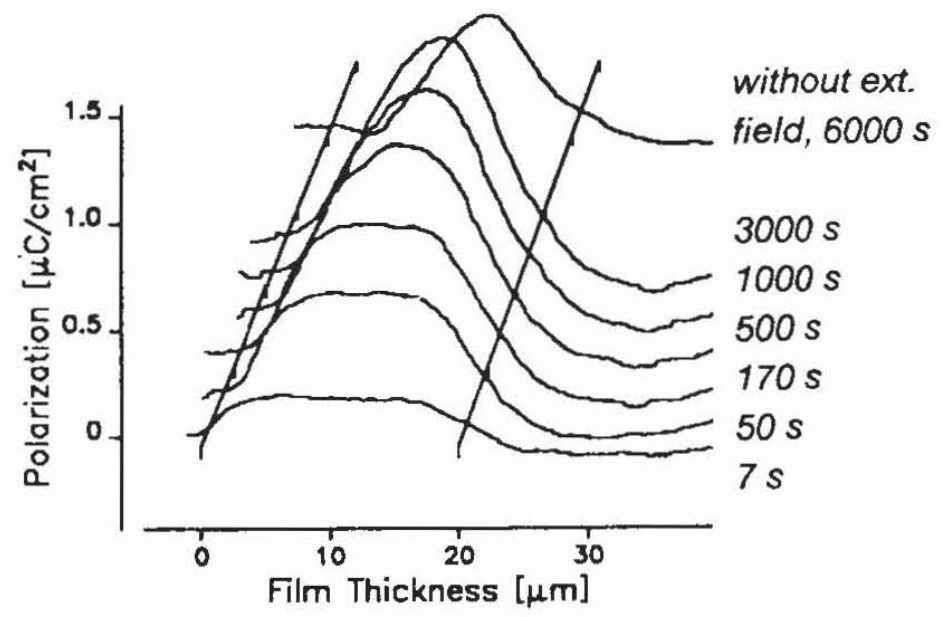

At medium field strength $(0.8 \mathrm{MV} / \mathrm{cm})$, see Fig.2b, the profile shows a nearly rectangular shape. The zone near the cathode, which is free from polarization, is smaller than at lower field strength. At both electrodes again homo charge injection is observed. 
Fig. 2b:

Stretched film,

thickness $20 \mu \mathrm{m}$,

$\alpha / \beta$ phase ratio 3.9

field strength $0.8 \mathrm{MV} / \mathrm{cm}$

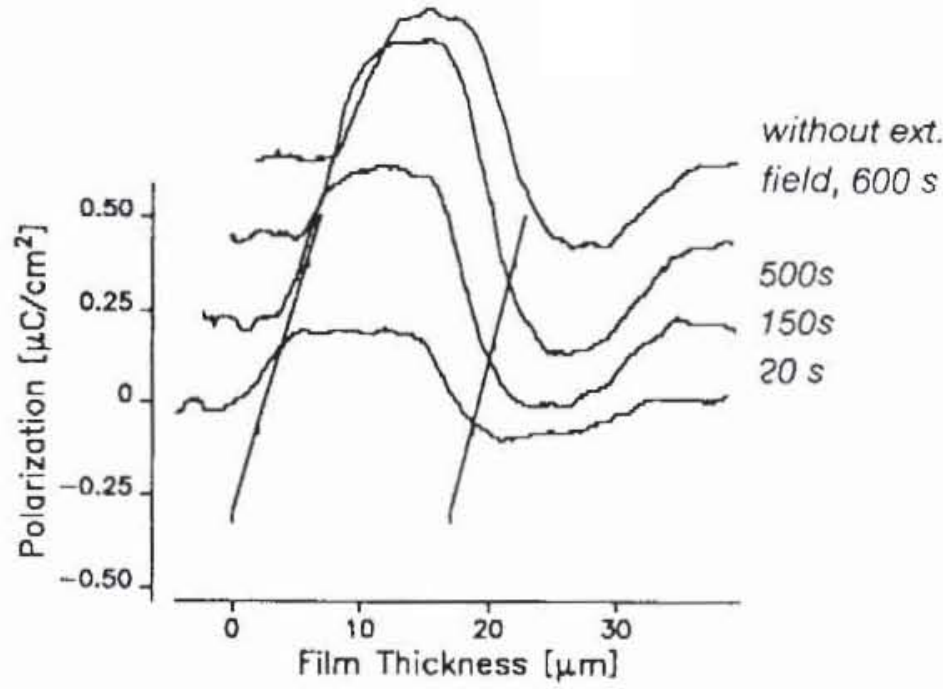

For higher field strength $(1.8 \mathrm{MV} / \mathrm{cm})$ homogeneous profiles are observed as depicted in Fig. 2c. In this case the transition from zero to maximum polarization at the film boundaries is within the experimental resolution.

Fig. 2c:

Stretched film, thickness $20 \mu \mathrm{m}$, $\alpha / \beta$ phase ratio 3.9 field strength $1.8 \mathrm{MV} / \mathrm{cm}$

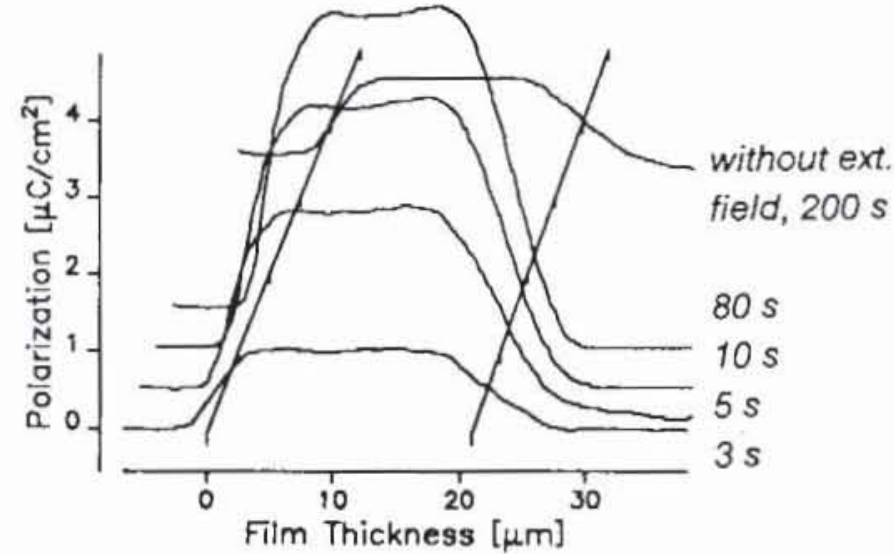

Now the stretched films were finally annealed at $180^{\circ} \mathrm{C}$ for $3 \mathrm{~h}$. The film thickness increased by a factor of 2.5 . The $\alpha / \beta$ phase ratio increased to 17 . The polarization develops again only in a small region close to the anode. The polarization step at the

Fig. 3:

Annealed film, thickness $55 \mu \mathrm{m}$, $\alpha / \beta$ phase ratio 17 field strength $0.6 \mathrm{MV} / \mathrm{cm}$

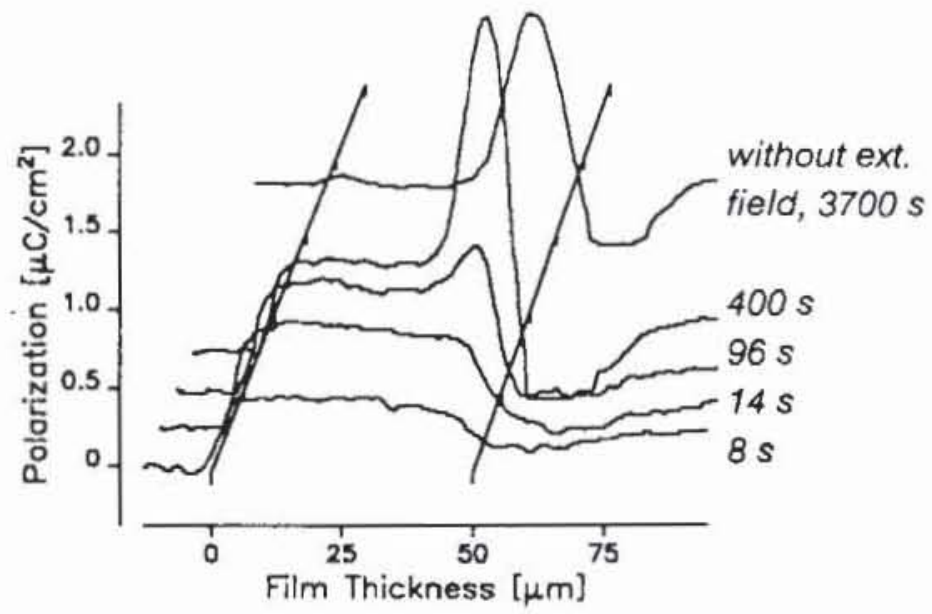


cathode gets smoothed out with time indicating homo charge injection. The plateau between the cathode and the anode polarization peak is decreasing with increasing polarization in the peak, indicating an increasing internal field in the polarization zone and charge trapping at the boundaries of this zone.

For comparison we investigated also a stretched material supplied from the Kureha company, Japan. The $\alpha / \beta$ ratio was determined to 1.25 . Fig.4a shows the time evolution of the polarization for this sample under an external field of $0.6 \mathrm{MV} / \mathrm{cm}$. Again a central polarization zone develops for this $\beta$-material.

Fig. 4a:

Kureha, as received thickness $50 \mu \mathrm{m}$, $\alpha / \beta$ phase ratio 1.25 field strength $0.6 \mathrm{MV} / \mathrm{cm}$

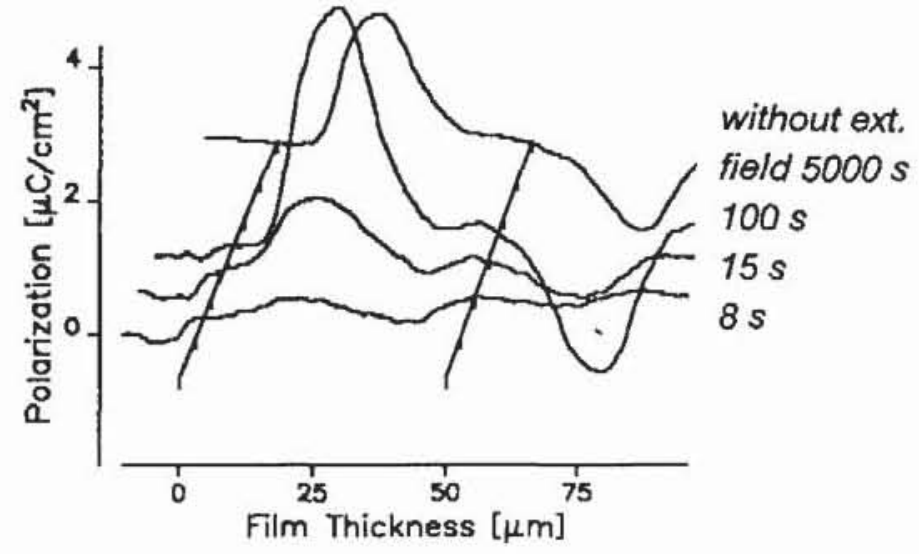

Now this film material was annealed at $160^{\circ} \mathrm{C}$ for $3 \mathrm{~h}$. The $\alpha / \beta$ ratio increased to only 1.5. The thickness increased by $10 \%$ to $55 \mu \mathrm{m}$. The polarization development in Fig. $4 \mathrm{~b}$ shows a polarization zone shifted towards the anode. First the step at the cathode becomes smoothed indicating charge injection. The same occurs later at the anode. The position of the resulting internal permanent polarization apparently is determined by the differences in the charge mobility and injection from the electrodes.

Fig. 4b:

Annealed Kureha film, thickness $55 \mu \mathrm{m}$, $\alpha / \beta$ phase ratio 1.5 ficld strength $0.6 \mathrm{MV} / \mathrm{cm}$

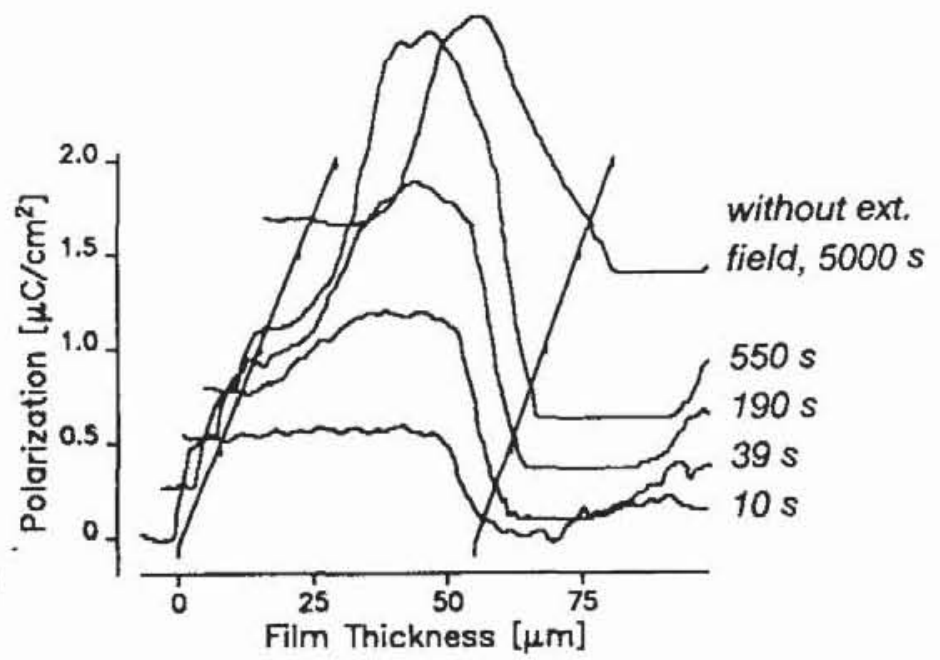

\section{Summary and Conclusions}

The development of inhomogeneous and internal polarization zones can be attributed to charge injection and charge trapping at the polarization zone boundaries $/ 3,9 /$. The polarization zone develops at a position where the critical field strength for dipole 
orientation in the crystallites is exceeded by the approach of injected homo charges (or without injection by the depletion of internal homocharges and the excess of heterocharges). The critical field for $\alpha$-crystallites corresponds to an electric field phase transition at $1.2 \mathrm{MV} / \mathrm{cm} / 10 /$. (For $\beta$-material a thin film permanent polarization threshold of $0.5 \mathrm{MV} / \mathrm{cm}$ is reported $/ 12 /$.)

Since we observe for $\alpha$-material a polarization zone at the anode for a total field strength of $0.6 \mathrm{MV} / \mathrm{cm}$, this indicates strong negative charge injection at the cathode with high charge mobility and little positive charge injection from the anode. There may be also an additional field enhancement in the anode regime by intrinsic positive charges in the film which are depleted at the anode by extraction to the cathode leaving excess heterocharges in the anode regime $/ 11 /$.

The development of 'central polarization zones in PVDF containing $\beta$-crystallites indicates injection of charges with both signs and almost equal mobility. Since the difference in the position of the polarization zone between pure $\alpha$-material and films containing $\beta$-crystallites evidently is caused by the $\beta$-crystallite content, the $\beta$-crystallites either determine the charge injection rate or the mobility or both. A strong reduction of the mobility of negative (and positive) charges can be explained by the model of polarized crystallites as traps $/ 3,9 /$. The trap properties of $\beta$-crystallites can be explained by the same mechanism of charge trapping by oriented dipole ends at the crystallite surface. Thus the transition from the polarization zone at the anode in $\alpha$-material to the central polarization in $\beta$-material can be explained by the reduction of the mobility of negative charges by $\beta$-crystallites. The influence on the mobility of positive charges is expected to be comparatively small, because this mobility appears to be already low in the pure $\alpha$-material.

\section{Acknowledgement}

The authors are very grateful to A.Dörnen for the IR absorption measurements. Financial support by the Deutsche Forschungsgemeinschaft is gratefully acknowledged.

\section{$\underline{\text { 5. References }}$}

1. P.J.Phelan et al., Ferroelectrics 7,375 (1974);

2. H.Sussner, K.Dransfeld, J.Poly.Sci.: Polymer Physics Ed., 16,529 (1978)

3. W.Eisenmenger, M.Haardt, Solid State Comm., 41, 917 (1982)

4. S.B.Lang et al., J.Appl.Phys., 54, 5598 (1983)

5. G.M.Sessler et al., IEEE 1987 Annual Report CEIDP, 319 (1987)

6. for a review see: G.M.Sessler (ed.): Electrets, Springer (1987)

7. K.Holdik,W.Eisenmenger, ISE5, Heidelberg, p. (1985)

8. N. Murayama, J.Poly.Sci.: Polymer Physics Ed., 13, 929 (1975)

9. W.Eisenmenger et al., IEEE 1982 Annual Report CEIDP, 52 (1982)

10. G.T.Davies et al., J.Appl.Phys., 49, 4998 (1978)

11. K.Holdik, Thesis, Universität Stuttgart, 1985

12. D.Nägele, D.Y.Yoon, Appl.Phys.Lett., 33, 132 (1978) 